\title{
罗丹明B诱导铈离子催化Belousov-Zhabotinsky 反应的振荡化学发光
}

段春凤, 刘光明, 张志锋, 崔华 ${ }^{*}$

中国科学技术大学化学系, 合肥 230026

*联系人, E-mail: hcui@ustc.edu.cn

2008-11-17 收稿, 2009-01-22 接受

中国科学院“引进海外杰出人才”计划和国家杰出青年科学基金(批准号: 20625517)资助项目

摘要 发现罗丹明 B 可诱导经典的铈离子催化的Belousov-Zhabotinsky(BZ)反应, 产生振荡的化学 发光. 这一新化学发光振荡体系, 即罗丹明 B-丙二酸-溴酸钾-硫酸铈铵-硫酸化学振荡体系, 具有 明显的双峰振荡特点. 反应物的初始浓度对振荡图形有显著的影响. 为了研究化学发光峰产生的 机理, 构建了一个通用型电压和发光同时检测的实验平台, 将该体系的电压振荡图形与发光振荡 图形进行了对照. 根据化学发光光谱、紫外可见吸收光谱和时间分辨苂光光谱的研究结果, 提出 了一个可能的化学发光机理. 初步认为两个发光峰分别与 Ce(IV)和 $\mathrm{Br}_{2}$ 氧化罗丹明 B 的中间产物 有关. 本工作为复杂的化学振荡反应提供了一个新的研究技术和方法.

关键词 化学发光 化学振荡 罗丹明 B 同时监测
Belousov-Zhabotinsky(BZ)反应是一个典型的液 相化学振荡反应, 其本质是在较强的硫酸介质中, 以 过渡金属离子 (如 $\mathrm{Ce}^{3+}-\mathrm{Ce}^{4+[1]}, \mathrm{Mn}^{2+}-\mathrm{Mn}^{3+[2]}$ )或者金属 离子配合物 (如 Ru(bpy) $)_{3}{ }^{2+}-\mathrm{Ru}(\mathrm{bpy})_{3}{ }^{3+[3]}, \mathrm{Fe}(\text { phen })_{3}{ }^{2+}-$ $\mathrm{Fe}(\mathrm{phen})_{3}{ }^{3+[4]}$ ) 为催化剂, 用溴酸盐氧化有机物(通常 为丙二酸)的反应. 以铈离子为催化剂的BZ反应, 可在 反应过程中观察到溶液颜色由黄变淡的周期性振荡现 象. 对于这一复杂化学反应的机理, 目前被人们普遍接 受的是Field等人 ${ }^{[5]}$ 提出的FKN机理. 该机理认为体系 中同时存在着 $\mathrm{Ce}^{4+} / \mathrm{Ce}^{3+}$ 和 $\mathrm{Br}^{-}$的振荡行为, 包含 10 个中 间反应步骤, 可以简单总结为以下三个过程:

过程 $\mathrm{A}$ ：当 $\mathrm{Br}^{-}$浓度较大时, $\mathrm{BrO}_{3}^{-}$被 $\mathrm{Br}^{-}$还原成 $\mathrm{Br}_{2}$, 反应式为

$$
\mathrm{BrO}_{3}^{-}+5 \mathrm{Br}^{-}+6 \mathrm{H}^{+} \rightarrow 3 \mathrm{Br}_{2}+3 \mathrm{H}_{2} \mathrm{O}
$$

生成的 $\mathrm{Br}_{2}$ 可与丙二酸反应生成 $\mathrm{BrCH}(\mathrm{COOH})_{2}$,

$$
\mathrm{CH}_{2}(\mathrm{COOH})_{2}+\mathrm{Br}_{2} \rightarrow \mathrm{BrCH}(\mathrm{COOH})_{2}+\mathrm{Br}^{-}+\mathrm{H}^{+}
$$

(1)和(2)组成了过程 $A$, 总反应式为

$$
\begin{gathered}
\mathrm{BrO}_{3}^{-}+2 \mathrm{Br}^{-}+3 \mathrm{CH}_{2}(\mathrm{COOH})_{2}+3 \mathrm{H}^{+} \\
\rightarrow 3 \mathrm{BrCH}(\mathrm{COOH})_{2}+3 \mathrm{H}_{2} \mathrm{O}
\end{gathered}
$$

$\mathrm{BrO}_{3}^{-}$可被 $\mathrm{Ce}^{3+}$ 还原, 同时 $\mathrm{Ce}^{3+}$ 被氧化为 $\mathrm{Ce}^{4+}$, 该过程 的总反应式为

$$
\mathrm{BrO}_{3}^{-}+4 \mathrm{Ce}^{3+}+5 \mathrm{H}^{+} \rightarrow \mathrm{HOBr}+4 \mathrm{Ce}^{4+}+2 \mathrm{H}_{2} \mathrm{O}
$$

过程 $\mathrm{C}$ : 由过程 $\mathrm{B}$ 产生的 $\mathrm{Ce}^{4+}$ 可以氧化 $\mathrm{BrCH}(\mathrm{COOH})_{2}$, 实现 $\mathrm{Br}^{-}$的再生, 总反应式如反应式 (5). 由于该过程伴随 $\mathrm{Ce}^{4+}$ 到 $^{\mathrm{Ce}^{3+}}$ 的转化, 因此可以观 察到溶液的颜色由黄色到无色的变化. 如果采用电 压检测法, 可以观察到溶液电压的降低.

$$
\begin{aligned}
4 \mathrm{Ce}^{4+} & +\mathrm{BrCH}(\mathrm{COOH})_{2}+\mathrm{HOBr}+\mathrm{H}_{2} \mathrm{O} \\
& \rightarrow 2 \mathrm{Br}^{-}+4 \mathrm{Ce}^{3+}+3 \mathrm{CO}_{2}+6 \mathrm{H}^{+}
\end{aligned}
$$

经由过程 $\mathrm{C}$, 当 $\mathrm{Br}^{-}$浓度恢复到一定水平时, 体 系便回到过程 A, 如此形成周期性振荡. 从宏观上看, 整个 BZ 反应的过程就是溴酸盐氧化丙二酸的过程, 总反应式为

$$
\begin{aligned}
& 2 \mathrm{BrO}_{3}^{-}+3 \mathrm{CH}_{2}(\mathrm{COOH})_{2}+2 \mathrm{H}^{+} \\
& \rightarrow 2 \mathrm{BrCH}(\mathrm{COOH})_{2}+3 \mathrm{CO}_{2}+4 \mathrm{H}_{2} \mathrm{O}
\end{aligned}
$$

这一复杂化学体系的机理研究在很大程度上依 赖于对体系中单一或者多个参数的监测方法. 比如 FKN机理的提出主要基于对体系中 $\mathrm{Br}^{-}$浓度的监测. 目前, 观测BZ反应的方法包括电化学 ${ }^{[6]}$ 、紫外可见吸 
收 ${ }^{[7]}$ 、苂光 ${ }^{[8]}$ 和化学发光 (chemiluminescence, CL) ${ }^{[9]}$. 其中, 化学发光法在液相化学振荡中的应用较少. 目 前报道的振荡化学发光体系还仅仅局限于鲁米诺和 联吡啶钉化学发光体系. 前者用于 $\mathrm{CuSO}_{4}-\mathrm{KSCN}$ $\mathrm{H}_{2} \mathrm{O}_{2}$ 振荡反应的研究 ${ }^{[10]}$, 后者用于联吡啶钉催化的 $\mathrm{BZ} 反$ 应的监测 ${ }^{[9]}$.

最近, $\mathrm{Ma}$ 等人 ${ }^{[11]}$ 报道在硫酸介质中 $\mathrm{Ce}^{4+}$ 氧化罗 丹明B的反应可以产生化学发光. 因此, 在铈离子催 化的BZ反应中加入罗丹明B，就可能诱导这一经典 $\mathrm{BZ}$ 反应产生化学发光. 在本项工作中, 我们发现对 于经典的丙二酸-溴酸钾-硫酸铈铵-硫酸这一BZ反应, 在没有罗丹明B存在时没有化学发光现象, 但加入罗 丹明 $\mathrm{B}$ 后, 可以观察到振荡化学发光现象, 并且与其 他的振荡化学发光体系明显不同, 它具有双峰振荡 的显著特点. 我们建立了一个能够同时进行电势和 化学发光检测的仪器平台, 对振荡过程进行了实时 监测和比对. 同时利用紫外吸收光谱和苂光光谱技 术对反应的中间产物和最终产物进行了表征. 在此 基础上, 提出了产生化学发光峰的可能机理.

\section{1 实验}

（i ）试剂. 实验用水为二次蒸馏水. 罗丹明 $B$ 、 溴酸钾、硫酸铈铵、硫酸(均为分析纯)和丙二酸 (化学 纯)均购自上海化学试剂公司. 其储备液浓度分别为: $5.0 \times 10^{-3}, 0.20,4.0 \times 10^{-3}, 3.0,0.45 \mathrm{~mol} / \mathrm{L}$.

(ii ) 仪器. 实验采用的电位和化学发光同时检 测的仪器系统如图 1 所示, 主要包括两个部分. A 为 化学振荡反应部分, 其主要部件包括圆柱型玻璃反 应器 $(75 \mathrm{~mL}$ )、JB-1 搅拌器(上海精科雷磁)和 501 型超 级恒温槽(上海市实验仪器厂), 用于发生和控制 BZ 反应. B 为检测部分, 包括一个圆柱型发光池、 CR105 光电倍增管(北京滨松电子有限公司)、GD-1 微光测量仪(西安瑞迈电子科技有限公司)和 N-2000 双通道色谱工作站(浙江大学智能信息工程研究所). $\mathrm{A}$ 和 $\mathrm{B}$ 部分通过蠕动泉和管路(西安瑞迈电子科技有 限公司)连接. 实验进行时, 将 A 中反应器里的溶液 通过蠕动洜吸入 $\mathrm{B}$ 中的发光池中, 然后回到反应器 中, 形成一个封闭的回路. 电位检测通过固定在发光 池上的铂丝和银丝进行, 化学发光检测则由发光池 旁边的光电倍增管进行, 两种信号同时被工作站采 集并记录. 当只采集化学发光信号时, 可以将 $\mathrm{B}$ 中的 圆柱型发光池改为螺旋型以获得更强的发光信号.

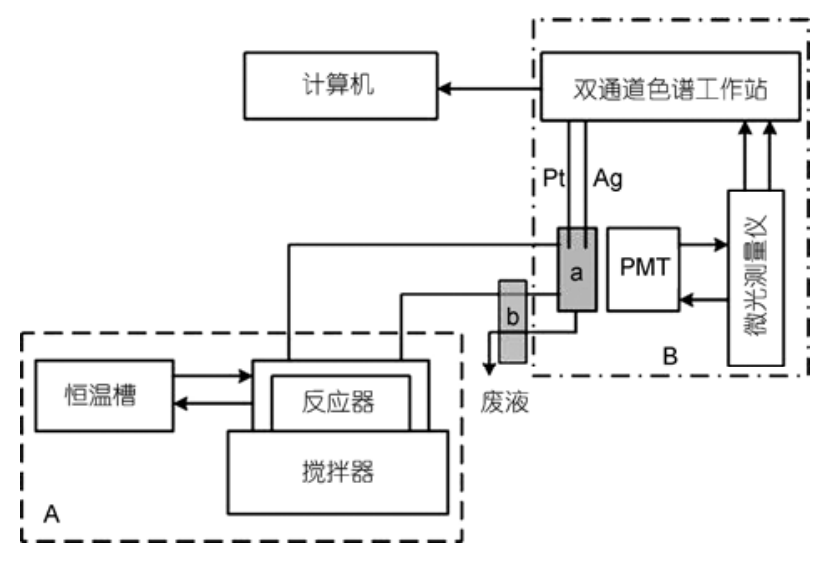

图 1 电压和化学发光同时检测化学振荡仪器示意图 $\mathrm{A}$ 为化学振荡反应部分, $\mathrm{B}$ 为检测部分. $\mathrm{a}$ 为发光池, $\mathrm{b}$ 为蠕动意. PMT 为光电倍增管, 高压: $-750 \mathrm{~V}$; 原转速: $60 \mathrm{r} / \mathrm{min}$

苂光光谱在RF-5301PC 苂光光度计(Shimadzu, Japan)上进行. 紫外可见吸收光谱在UV-2401 PC 分 光度计(Shimadzu, Japan)上进行. 通过在光电倍增管 和发光池之间插入 300 700 $\mathrm{nm}$ 截止滤光片, 可以获 得各个波长区间的化学发光强度, 从而获得化学发 光光谱 ${ }^{[12]}$.

(iii) 实验方法. 在反应器中依次加入丙二酸、 蒸馏水、硫酸、溴酸钾, 最后同时加入罗丹明 $\mathrm{B}$ 和硫 酸铈铵, 总体积固定为 $50 \mathrm{~mL}$.

\section{2 结果与讨论}

\section{1 振荡化学发光现象}

在反应器中预先依次加入丙二酸、硫酸和溴酸钾, 当罗丹明 $\mathrm{B}$ 和硫酸铈铵同时加入反应器时可以观察 到振荡化学发光现象如图 2 所示, 在一个周期内可以 观察到两个发光峰(CL-I 和 CL-II), 在振荡开始之前 的诱导期能观察到一个宽发射峰(CL-III). 发光峰 CLI 的光强在振荡初期逐渐增大, 之后随时间变化非 常缓慢, 而发光峰 CL-II 的光强衰减很快, 表明两个 发光峰在振荡过程中的发光途径不同. 在没有补充 初始反应物的条件下, 振荡的寿命长达 $90 \mathrm{~min}$ 以上. 同时, 振荡周期、振幅(化学发光强度)和振荡图形受反 应物的初始浓度影响很大. 比如, 罗丹明 $\mathrm{B}$ 初始浓度的 不同会导致化学发光振荡图形发生明显变化. 如图 3 所 示, 当罗丹明 B 的初始浓度较小时(如 $0.1 \mathrm{mmol} / \mathrm{L}$ ), 观 察不到 CL-II; 随着浓度的不断增大, CL-II 越来越明 显, 同时诱导期越来越长, 表明 CL-II 的发光与罗丹 
明 B 相关. 类似地, CL-I 和 CL-II 的振荡行为也随其 他反应物的初始浓度变化而变化.

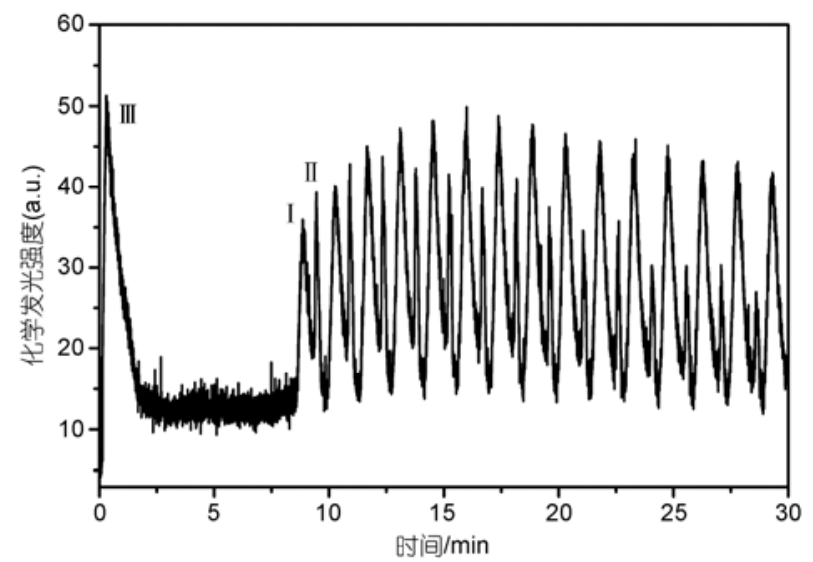

图 2 罗丹明 B-丙二酸-溴酸钾-硫酸铈铵-硫酸体系的振荡 化学发光图

实验条件: $5.0 \times 10^{-4} \mathrm{~mol} / \mathrm{L}$ 罗丹明 $\mathrm{B} ; 9.0 \times 10^{-2} \mathrm{~mol} / \mathrm{L}$ 丙二酸; $4.0 \times 10^{-2} \mathrm{~mol} / \mathrm{L}$ 溴酸钾; $8.0 \times 10^{-4} \mathrm{~mol} / \mathrm{L}$ 硫酸铈铵; $0.60 \mathrm{~mol} / \mathrm{L}$ 硫酸; 螺旋形发光池; 室温

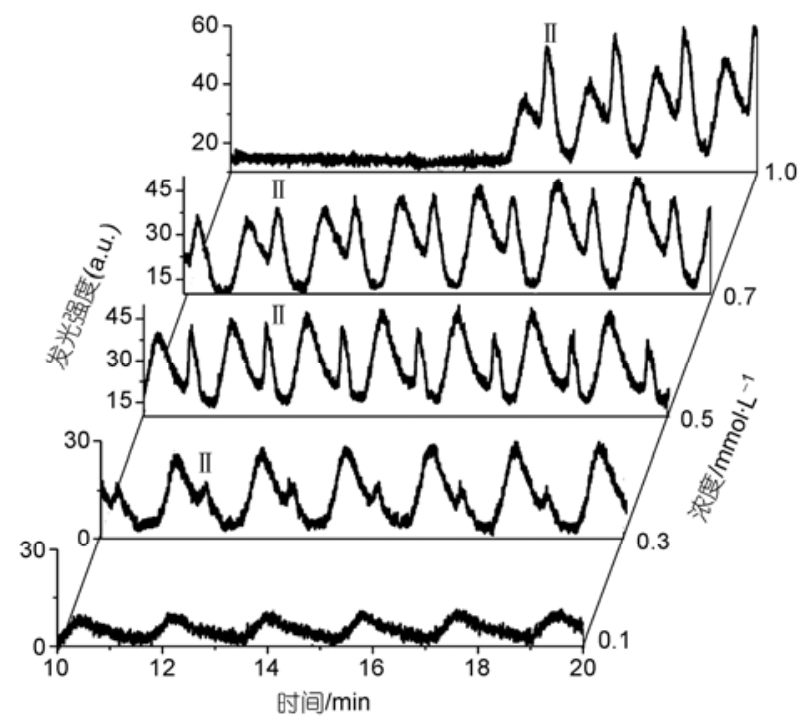

图 3 罗丹明 B 初始浓度对振荡化学发光的影响

实验条件: $9.0 \times 10^{-2} \mathrm{~mol} / \mathrm{L}$ 丙二酸; $4.0 \times 10^{-2} \mathrm{~mol} / \mathrm{L}$ 溴酸钾; $8.0 \times 10^{-4}$ $\mathrm{mol} / \mathrm{L}$ 硫酸铈铵; $0.60 \mathrm{~mol} / \mathrm{L}$ 硫酸; 螺旋形发光池; 室温

我们还采用电位和化学发光同时检测的方法观 察该振荡体系. 如图 4 所示, 每个周期内有一个电位 振荡峰, 两个发光峰对应于不同的电位. CL-I 对应 于电位下降的阶段, CL-II 对应于电位最低点附近. 根据FKN机理 ${ }^{[5]}$, 在电位下降阶段主要发生的是 $\mathrm{Ce}^{4+}$
被还原为 $\mathrm{Ce}^{3+}$ 的反应 (过程 $\mathrm{C}$ ), 而当电位处于最低点 附近时由于 $\mathrm{Br}^{-}$浓度的回升, 此时 $\mathrm{Br}^{-}$可以被溴酸盐 氧化生成 $\mathrm{Br}_{2}$ (过程 $\left.\mathrm{A}\right)$. 由于 $\mathrm{Ce}^{4+}$ 氧化丙二酸的反应不 会产生化学发光, 因此, CL-I 很可能跟 $\mathrm{Ce}^{4+}$ 与罗丹明 $\mathrm{B}$ 之间的反应相关, 而CL-II 则可能与 $\mathrm{Br}_{2}$ 的氧化还原 反应相关.

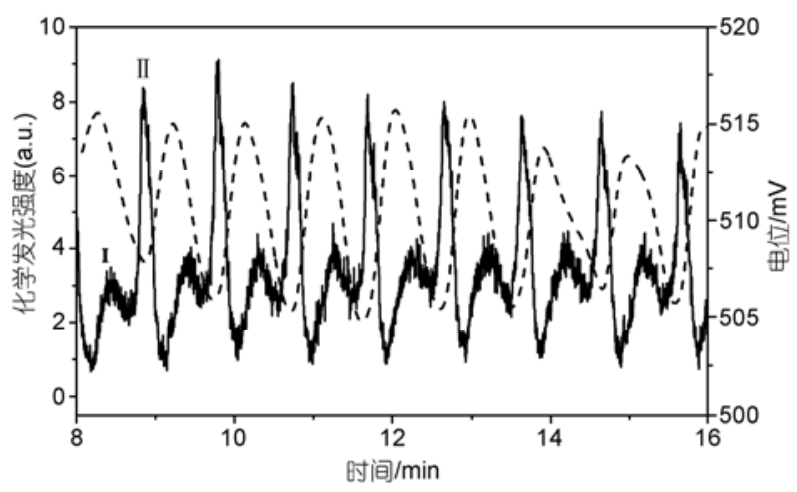

图 4 化学发光(实线)和电压(虚线)同时检测罗丹明 B丙二酸-溴酸钾-硫酸铈铵-硫酸振荡体系

实验条件：初始浓度同图 2; 圆柱形发光池; 室温

\section{2 化学发光光谱研究}

通过调节反应物的浓度可使化学振荡模式具有 较强的发光强度, 由此可以测量发光峰的化学发光 光谱. 如图 5(a)所示, 在这一模式下, CL-I 具有较高 的强度, 而发光峰 CL-II 变成了 CL-I 的肩峰(CL-I 和 CL-II 可经过电位检测进行确认识别), 同时, CL-III 分 裂为两个峰 $(a$ 和 $b)$. 在这种模式下检测到 CL-I 的发 光峰位于约 $490 \mathrm{~nm}$, 同时在约 $550 \mathrm{~nm}$ 附近有一肩峰. 由于 CL-II 与 CL-I 的严重重叠, CL-II 的化学发光光 谱与 CL-I 几乎相同, 只是在约 $550 \mathrm{~nm}$ 处的发光更为 明显. 由于很难得到一个 CL-II 发光很强而 CL-I 发 光很弱的化学发光振荡模式, 因此目前仍无法得到单 - CL-II 发光峰的化学发光光谱. 但是考虑到在这一 模式下 CL-II 的发光强度比 CL-I 低(图 5(a)), 而其光 谱图中约 $550 \mathrm{~nm}$ 处的发光却比 CL- I 更明显, 我们推 测 $550 \mathrm{~nm}$ 附近的发射主要来源于 CL-II. 为了验证这 一推论, 我们对图 2 所示的振荡化学发光采用 $510 \mathrm{~nm}$ 截止滤光片进行过滤. 结果发现, CL- I 消失而 CL- II 仍 然存在, 表明发光峰 CL-I 的发射低于 $510 \mathrm{~nm}$, 而发 光峰 CL-II 的发射高于 $510 \mathrm{~nm}$. 因此, $550 \mathrm{~nm}$ 附近的 发射来源于 CL-II, CL-I 的发射位于约 $490 \mathrm{~nm}$.

峰 CL-III a 和 CL-III b 的化学发光光谱如图 5(d)所 

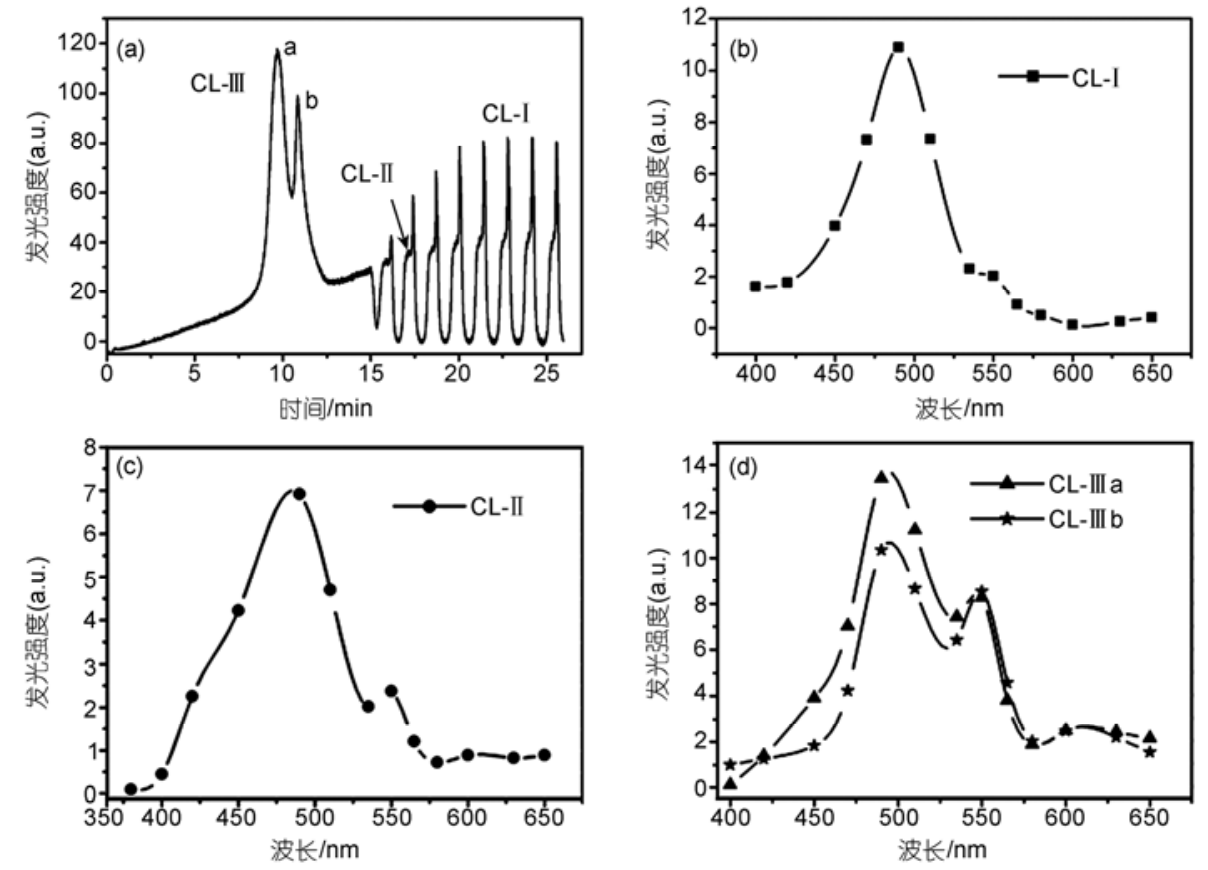

图 5 化学发光振荡图(a)和发光峰 CL-I (b), CL-II (c), CL-III (d)的化学发光光谱

实验条件: $5.0 \times 10^{-4} \mathrm{~mol} / \mathrm{L}$ 罗丹明 B; $9.0 \times 10^{-2} \mathrm{~mol} / \mathrm{L}$ 丙二酸; $1.2 \times 10^{-2}$ 溴酸钾; $8.0 \times 10^{-4} \mathrm{~mol} / \mathrm{L}$ 硫酸铈铵; $0.60 \mathrm{~mol} / \mathrm{L}$ 硫酸; 室温

示, 每个峰均在 3 个不同波长处有发射: 约 490, 550 和 $605 \mathrm{~nm}$, 前两者分别为 CL-I, CL-II 的发射波长, 605 $\mathrm{nm}$ 则为罗丹明 $\mathrm{B}$ 在实验条件下的苂光发射波长. 因 此, CL-III实际上是 3 种发光体 (CL-I 的发光体、CL-II 的发光体和罗丹明 B)化学发光的组合. 另一方面, 罗丹明 B 的发光(即 $605 \mathrm{~nm}$ 处的发射)只在诱导期出 现, 在化学发光振荡过程中(CL-I 和 CL-II )并未发现 罗丹明 B的发光, 说明在振荡开始之前罗丹明 B 很可 能已经完全消耗.

\section{3 产物的光谱表征}

为了确认体系的发光体, 采用了光谱技术对体 系的中间产物进行表征. 如图 6 所示, 在硫酸介质中 当罗丹明B与 $\mathrm{Ce}^{4+}$ 混合后, 其约 $555 \mathrm{~nm}$ 处的吸收降低, 而当溴酸钾溶液与罗丹明 $\mathrm{B}$ 混合或者与罗丹明 $\mathrm{B}+\mathrm{Ce}^{4+}$ 混合后, 约 $555 \mathrm{~nm}$ 处的吸收消失, 在约 $538 \mathrm{~nm}$ 处出现 一个新的吸收峰. 这些结果表明, 溴酸钾比 $\mathrm{Ce}^{4+}$ 更容 易氧化罗丹明 $\mathrm{B}$, 并且反应后得到一个新产物. 由于 $538 \mathrm{~nm}$ 处的吸收正好与罗丹明 $\mathrm{B}$ 的去乙基化产物 $N, N, N^{\prime}$-三乙基罗丹明的吸收吻合 ${ }^{[13]}$, 因此我们推测 在罗丹明 $\mathrm{B}$ 的氧化过程中发生了去乙基化.

图 7 显示了该振荡体系的时间分辨苂光光谱. 从 图 7(a)中可以看出, 当体系中没有溴酸钾时, 罗丹明

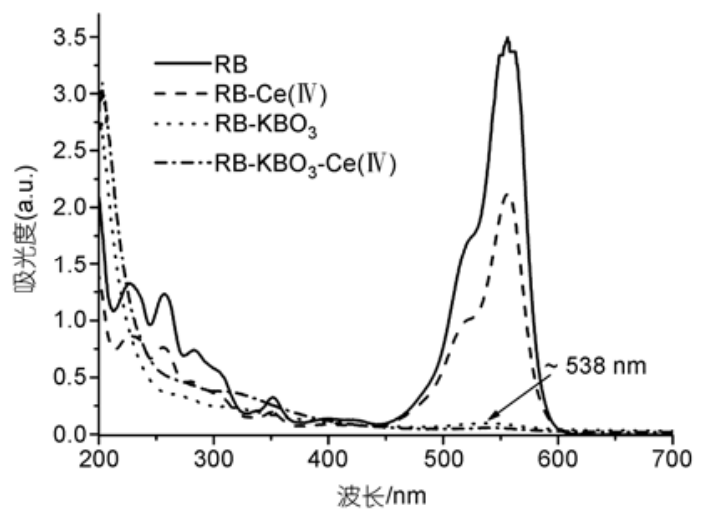

图 6 紫外可见吸收光谱

实验条件: $5.0 \times 10^{-4} \mathrm{~mol} / \mathrm{L}$ 罗丹明 B; $4.0 \times 10^{-2} \mathrm{~mol} / \mathrm{L}$ 溴酸钾; $8.0 \times 10^{-4} \mathrm{~mol} / \mathrm{L}$ 硫酸铈铵; $0.60 \mathrm{~mol} / \mathrm{L}$ 硫酸; 室温

B 在约 $605 \mathrm{~nm}$ 处的苂光发射强度随时间的变化几乎 保持恒定, 表明此条件下罗丹明 $\mathrm{B}$ 与 $\mathrm{Ce}^{4+}$ 的反应速度 很慢. 当体系中没有 $\mathrm{Ce}^{4+}$ 时, 约 $605 \mathrm{~nm}$ 处的苂光发 射消失, 而在 565 和 $474 \mathrm{~nm}$ 处出现两个新发射(图 7(b)).

结果表明罗丹明 $\mathrm{B}$ 更容易被溴酸钾氧化, 这与 紫外吸收光谱的结果一致. 位于 $565 \mathrm{~nm}$ 处的苂光峰 随时间逐渐偏移至 $555 \mathrm{~nm}$, 并且强度衰减很快(与发 光峰 CL- II 随时间的变化规律相同). 而 $474 \mathrm{~nm}$ 处的 

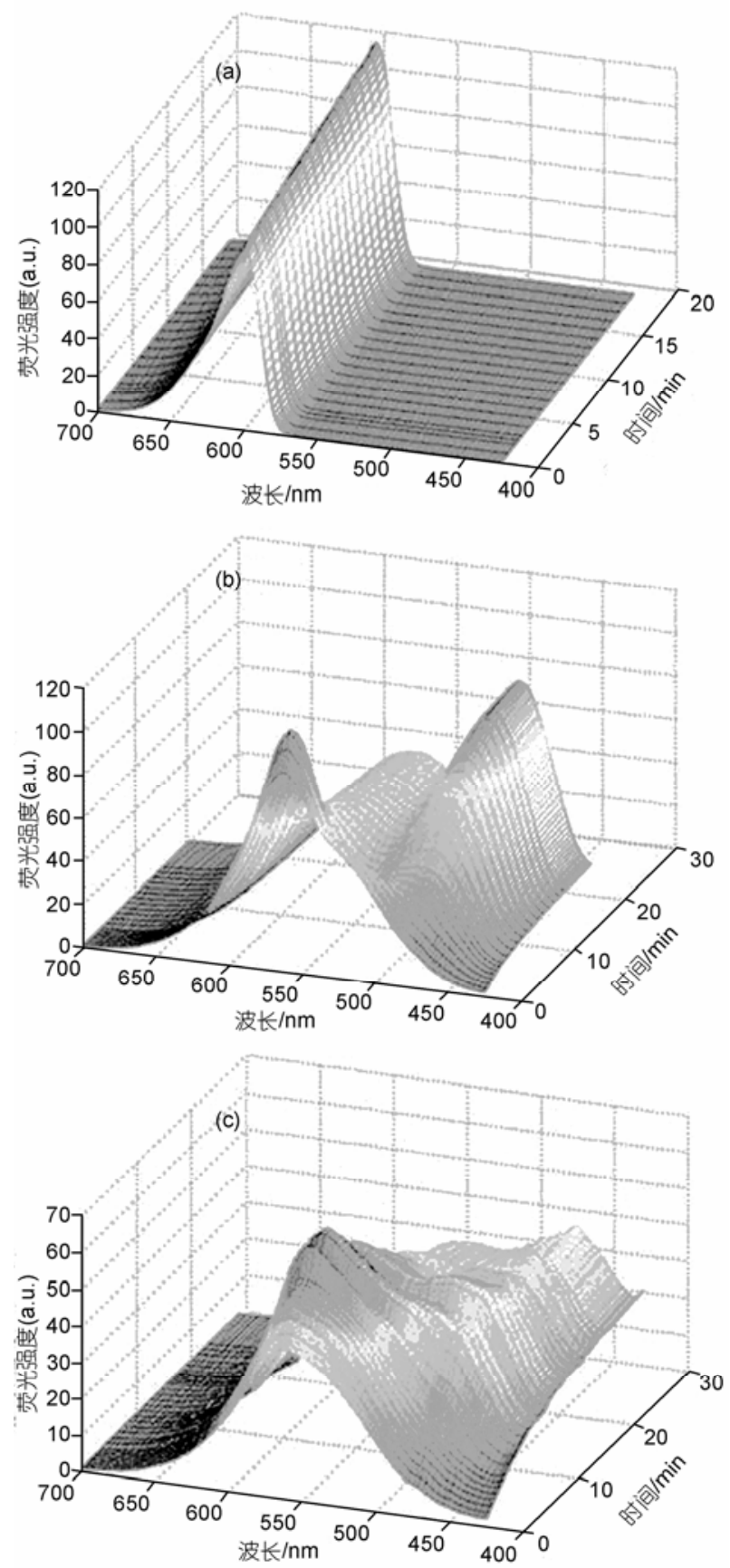

图 7 时间分辨苂光光谱

(a) 不含溴酸钾的体系: $5.0 \times 10^{-4} \mathrm{~mol} / \mathrm{L}$ 罗丹明 B; $9.0 \times 10^{-2} \mathrm{~mol} / \mathrm{L}$ 丙二酸; $8.0 \times 10^{-4} \mathrm{~mol} / \mathrm{L}$ 硫酸铈铵; $0.60 \mathrm{~mol} / \mathrm{L}$ 硫酸. (b) 不含硫酸 铈铵的体系: $5.0 \times 10^{-4} \mathrm{~mol} / \mathrm{L}$ 罗丹明 $\mathrm{B} ; 9.0 \times 10^{-2} \mathrm{~mol} / \mathrm{L}$ 丙二酸; $4.0 \times 10^{-2} \mathrm{~mol} / \mathrm{L}$ 溴酸钾; $0.60 \mathrm{~mol} / \mathrm{L}$ 硫酸. (c) 振荡体系: $5.0 \times 10^{-4}$ $\mathrm{mol} / \mathrm{L}$ 罗丹明 B; $9.0 \times 10^{-2} \mathrm{~mol} / \mathrm{L}$ 丙二酸; $4.0 \times 10^{-2} \mathrm{~mol} / \mathrm{L}$ 溴酸钾; $8.0 \times 10^{-4} \mathrm{~mol} / \mathrm{L}$ 硫酸铈铵; $0.60 \mathrm{~mol} / \mathrm{L}$ 硫酸

发射却随时间逐渐增强, 这与发光峰 CL- I 的振荡规 律不同. 当溴酸钾和 $\mathrm{Ce}^{4+}$ 同时存在, 即构成完整的振
荡体系时(图 7(c)), 同样可以观察到约 $605 \mathrm{~nm}$ 处菼 光峰的消失和两个新的苂光峰的出现(565 和 $474 \mathrm{~nm}$ ). 两个新苂光峰随时间的变化趋势与图 7(b)相同, 并且 具有振荡的特性. 这些结果表明, 555 565 nm 和约 $474 \mathrm{~nm}$ 处的两个苂光峰由溴酸钾氧化罗丹明 B 产生, 前者与发光峰 CL- II 明显相关, 因为他们具有相同的 发射波长和变化规律. 另一方面, 在振荡过程中没有 观察到罗丹明 $\mathrm{B}$ 的苂光发射, 说明在振荡开始之前 罗丹明 $\mathrm{B}$ 已经被溴酸钾完全消耗, 这与化学发光光 谱的结果吻合.

\section{4 机理讨论}

根据光谱表征结果, 罗丹明 $\mathrm{B}$ 在振荡开始之前已 经被溴酸钾完全消耗, 因此我们推测罗丹明 $\mathrm{B}$ 首先被 溴酸钾氧化生成一中间产物 $\mathrm{RB}_{\mathrm{ox} 1}$ ( $\lambda_{\mathrm{em}}$ 位于 550 565 $\mathrm{nm})$. 其次, 发光峰 CL- I 对应电压下降的阶段, 即 $\mathrm{Ce}^{4+}$ 被还原为 $\mathrm{Ce}^{3+}$ 的阶段, 因此我们推测 CL- I 是由 $\mathrm{RB}_{\mathrm{ox} 1}$ 与 $\mathrm{Ce}^{4+}$ 的反应产生. 而根据电压和发光同时检 测的结果, 发光峰 CL- II 可能与 $\mathrm{Br}_{2}$ 的氧化还原反应 相关. 根据文献报道 ${ }^{[14]}$, 在罗丹明 $6 \mathrm{G}$-溴酸盐化学发 光体系中, 罗丹明 $6 \mathrm{G}$ 被中间产生的 $\mathrm{Br}_{2}$ 氧化, 反应放 出的能量可以激发罗丹明 $6 \mathrm{G}$ 产生化学发光. 由于相 似的分子结构, 我们推测罗丹明 $\mathrm{B}$ 或者其中间的氧化 产物 $\mathrm{RB}_{\mathrm{ox} 1}$ 同样可以被 $\mathrm{Br}_{2}$ 氧化并被激发产生化学发 光. 因此, 发光峰CL-II 很可能由 $\mathrm{RB}_{\mathrm{ox} 1}$ 与 $\mathrm{Br}_{2}$ 的反应 产生. 可能的发光机理如下:

$$
\begin{gathered}
\mathrm{RB}+\mathrm{BrO}_{3}^{-} \rightarrow \mathrm{RB}^{*}+\mathrm{RB}_{\mathrm{ox} 1} \\
\mathrm{RB}^{*} \rightarrow \mathrm{RB}+h v(\sim 605 \mathrm{~nm}) \\
\mathrm{RB}_{\mathrm{ox} 1}+\mathrm{Ce}^{4+} \rightarrow \mathrm{Ce}^{3+}+\mathrm{RB}_{\mathrm{ox} 2}^{*} \\
\mathrm{RB}_{\mathrm{ox} 2}^{*} \rightarrow \mathrm{RB}_{\mathrm{ox2}}+h v(490 \sim 500 \mathrm{~nm}) \\
\mathrm{BrO}_{3}^{-}+4 \mathrm{Ce}^{3+}+5 \mathrm{H}^{+} \rightarrow \mathrm{HOBr}+4 \mathrm{Ce}^{4+}+2 \mathrm{H}_{2} \mathrm{O} \\
\mathrm{RB}_{\text {ox1 }}+\mathrm{Br}_{2} \rightarrow \mathrm{RB}_{\mathrm{ox3}}+\mathrm{Br}^{-}+\mathrm{RB}_{\mathrm{ox} 1}^{*} \\
\mathrm{RB}_{\mathrm{ox} 1}^{*} \rightarrow \mathrm{RB}_{\mathrm{ox1}}+h v(550 \sim 565 \mathrm{~nm}) \\
\mathrm{BrO}_{3}^{-}+5 \mathrm{Br}^{-}+6 \mathrm{H}^{+} \rightarrow 3 \mathrm{Br}_{2}+3 \mathrm{H}_{2} \mathrm{O}
\end{gathered}
$$

其中 $\mathrm{RB}, \mathrm{RB}^{*}, \mathrm{RB}_{\mathrm{ox} 1}, \mathrm{RB}_{\mathrm{ox} 2}, \mathrm{RB}_{\mathrm{ox} 1}^{*}$ 和 $\mathrm{RB}_{\mathrm{ox} 2}^{*}$ 代表罗丹 明 $\mathrm{B}$ 、它的中间氧化产物和相应的激发态. 在振荡开 始之前, 罗丹明 $\mathrm{B}$ 首先被溴酸钾氧化产生 $\mathrm{RB}_{\mathrm{ox} 1}\left(\lambda_{\mathrm{em}} \approx\right.$ $550 \sim 565 \mathrm{~nm})$, 同时形成激发态罗丹明 $\mathrm{B}\left(\mathrm{RB}^{*}\right)$. 中间 产物 $\mathrm{RB}_{\mathrm{ox} 1}$ 进一步与 $\mathrm{Ce}^{4+}$ 和 $\mathrm{Br}_{2}$ 反应, 分别形成激发 态 $\mathrm{RB}_{\mathrm{ox} 2}^{*}$ 和 $\mathrm{RB}_{\mathrm{ox} 1}^{*}$, 产生 490 500 nm 和 550 565 nm 两个发射带, 分别对应发光峰 CL-I 和 CL-II. 3 个发 光体共同形成了 CL- III 的 3 个发射峰. 发光反应(c)消 耗的 $\mathrm{Ce}^{4+}$ 可以经由过程 $\mathrm{B}$ (反应式(4))得到恢复, 而发 光反应(e)消耗的 $\mathrm{Br}_{2}$ 则可经过过程 $\mathrm{A}$ (反应式(1))得到 
恢复, 由此形成化学发光的周期性振荡. 由于很难获 得更多有关罗丹明 $\mathrm{B}$ 的中间氧化产物 $\left(\mathrm{RB}_{\mathrm{ox} 1}\right.$ 和 $\left.\mathrm{RB}_{\mathrm{ox} 2}\right)$ 的光谱信息，如质谱和核磁共振光谱，因此目前还无 法鉴定它们的化学结构.

\section{3 结论}

本文发现了一个新化学发光振荡体系，即罗丹 明 B-丙二酸-溴酸钾-硫酸铈铵-硫酸振荡体系. 通过 以罗丹明 $\mathrm{B}$ 为指示剂, 可以对 $\mathrm{Ce}^{4+}$ 催化的经典 $\mathrm{BZ}$ 反 应进行化学发光检测. 与报道的其他化学发光振荡 体系不同, 该体系具有明显的双峰振荡的特点. 通过 对化学发光光谱、吸收和苂光发射光谱的研究，提出 了一种可能的发光机理, 推测发光峰 CL-I 和 CL-II
分别由 $\mathrm{Ce}^{4+}$ 和 $\mathrm{Br}_{2}$ 氧化罗丹明 $\mathrm{B}$ 的中间产物导致. 因 此, 两个振荡发光峰直接或者间接地反映了 $\mathrm{Ce}^{4+} /$ $\mathrm{Ce}^{3+}$ 和 $\mathrm{Br}^{-}$的振荡行为, 与FKN机理吻合, 表明化学 发光检测技术可以用于化学振荡反应的研究. 该工 作中建立的电压和发光同时检测的实验平台具有通 用性, 并且易于改装升级为结合其他光谱技术(如紫 外, 苂光)的多功能检测平台, 为化学振荡这一复杂 化学体系的机理研究提供了一个新的实验技术和研 究方法. 另一方面, 这个新化学发光振荡体系具有寿 命长(>90 min), 诱导期短 $(<10 \mathrm{~min})$ 的特点, 适合应 用脉冲干扰技术建立分析方法 ${ }^{[15]}$, 同时由于化学发 光具有灵敏度高的特点, 因此有望利用该体系建立 基于化学振荡的高灵敏度检测方法.

\section{参考文献}

1 Field R J, Schneider F W. Oscillating chemical reactions and nonlinear dynamic. J Chem Educ, 1989, 66: 195-204

2 Franck U F. Chemical oscillations. Angew Chem Int Edn Engl, 1978, 17: 1-15 [doi]

3 Weigt H R. Chemiluminescence oscillations driven by a flow-through reactor in the $\left[\mathrm{Ru}(\mathrm{bpy})_{3}\right]^{2+}$ catalyzed Belousov-Zhabotinskii reaction. Angew Chem Int Edn Engl, 1992, 31: 355-357[doi]

4 Yatsimirskii K B, Strizhak P E, Ivaschenko T S. Potential of chaotic chemical systems in nanotrace analysis based on the Belousov-Zhabotinskii reaction ( $\mathrm{BrO}_{3}^{-}$-malonic acid-ferroin). Determination of manganese ( II ). Talanta, 1993, 40: 1227-1232 [doi]

5 Field R J, Körös E, Noyes R M. Oscillations in chemical systems. II. Thorough analysis of temporal oscillation in the bromate-cerium-malonic acid system. J Am Chem Soc, 1972, 94: 8649-8664[doi]

6 Orbán M. Oscillations and bistability in the $\mathrm{Cu}$ (II )-catalyzed reaction between $\mathrm{H}_{2} \mathrm{O}_{2}$ and KSCN. J Am Chem Soc, 1986, 108: 68936898[doi]

7 Bolletta F, Prodi L, Zaccheroni N. Oscillating luminescence in the Belousov-Zhabotinsky reaction catalyzed by Ru(bpy) ${ }_{3}{ }^{2+}$. Inorg Chim Acta, 1995, 233: 21-23 [doi]

8 Bolletta F, Prodi L, Zaccheroni N. Oscillating photoluminescence in the cerium ion catalyzed Belousov-Zhabotinsky reaction. Chem Phys Lett, 1995, 237: 346-348[doi]

9 Bolletta F, Balzani V. Oscillating chemiluminescence from the reduction of bromate by malonic acid catalyzed by tris(2,2'-bipyridine)ruthenium( II ). J Am Chem Soc, 1982, 104: 4250-4251[doi]

10 Amrehn J, Resch P, Schneider F W. Oscillating chemiluminescence with luminol in the continuous flow stirred tank reactor. J Phys Chem, 1988, 92: 3318-3320[doi]

11 Ma Y J, Jin X Y, Zhou M, et al. Chemiluminescence behavior based on oxidation reaction of rhodamine B with cerium(IV) in sulfuric acid medium. Anal Chim Acta, 2003, 489: 173-181 [doi]

12 Cui H, Zou G Z, Lin X Q. Electrochemiluminescence of luminol in alkaline solution at a paraffin-impregnated graphite electrode. Anal Chem, 2003, 75: 324-331 [doi]

13 Wu T X, Liu G M, Zhao J C, et al. Photoassisted degradation of dye Pollutants. V. Self-photosensitized oxidative transformation of Rhodamine $B$ under visible light irradiation in aqueous $\mathrm{TiO}_{2}$ dispersions. J Phys Chem B, 1998, 102: 5845-5851 [doi]

14 Li B X, Liu M L, Zhang Z J, et al. Flow-injection chemiluminescence determination of formaldehyde with a bromate-rhodamine $6 \mathrm{G}$ system. Anal Sci, 2003, 19: 1643-1646[1doi]

15 高锦章. 化学振荡现象在分析化学中的应用. 西北师范大学学报, 2002, 38(3): 100-105 